AN ADAPTIVE METHOD WITH RIGOROUS ERROR CONTROL FOR THE HAMILTON-JACOBI EQUATIONS.

PART II: THE TWO-DIMENSIONAL STEADY STATE CASE

By

Bernardo Cockburn

and

Bayram Yenikaya

IMA Preprint Series \# 1994

( September 2004)

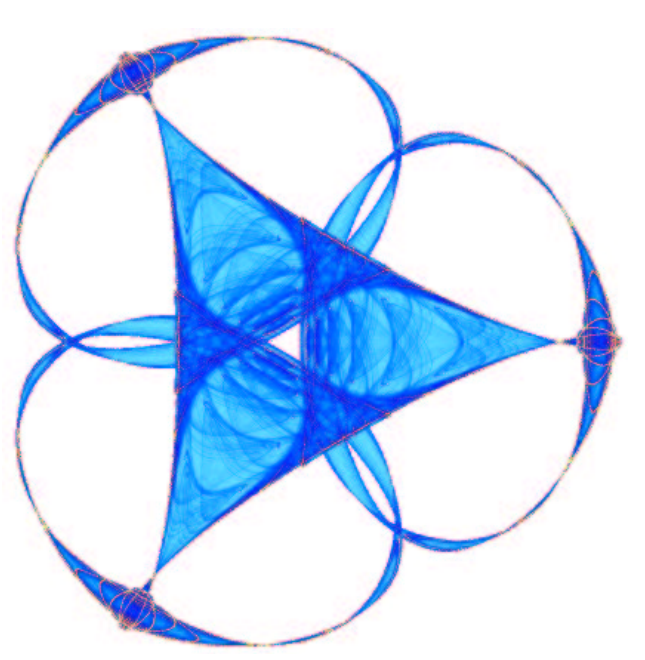

INSTITUTE FOR MATHEMATICS AND ITS APPLICATIONS

UNIVERSITY OF MINNESOTA

514 Vincent Hall

206 Church Street S.E.

Minneapolis, Minnesota 55455-0436

Phone: 612/624-6066 Fax: 612/626-7370

URL: http://www.ima.umn.edu 


\title{
An adaptive method with rigorous error control for the Hamilton-Jacobi equations. Part II: The two-dimensional steady state case
}

\author{
Bernardo Cockburn, Bayram Yenikaya
}

September 28, 2004

\begin{abstract}
In this paper, we devise and study an adaptive method for finding approximations to the viscosity solution of Hamilton-Jacobi equations. The method, which is an extension to two space dimensions of a similar method previously proposed for one space dimension, is studied in the framework of steady state Hamilton-Jacobi equations with periodic boundary conditions. It seeks numerical approximations whose $L^{\infty}$-distance to the viscosity solution is no bigger than a prescribed tolerance. A thorough numerical study is carried out which shows that a strict error control is achieved and that the method exhibits an optimal computational complexity which does not depend on the value of the tolerance or on the type of Hamiltonian.
\end{abstract}

\section{Introduction}

In this paper, we continue the study of adaptive methods for HamiltonJacobi equations began in [4] for the one dimensional case and extend it to two-dimensional problems of the same type. We present an efficient adaptive method for approximating the viscosity solution of the following model steady-state Hamilton-Jacobi equation

$$
u+H(\nabla u)=f \quad \text { in } \Omega=(0,1) \mathrm{x}(0,1),
$$

with periodic boundary conditions. For any given positive parameter $\tau$, the method obtains an approximation $u_{h}$ satisfying the quality constraint

$$
\left\|u-u_{h}\right\|_{L^{\infty}\left(\Omega_{h}\right)} \leq \tau,
$$


where $\Omega_{h}$ is the set of nodes on which the approximate solution is computed. We show the efficiency and the reliability of the method through extensive numerical experiments.

To the knowledge ot the authors, the method we present in this paper is the only adaptive algorithm for Hamilton-Jacobi equations in two dimensions. For related work, see [4] and the references therein. Let us briefly describe its main components. To find an approximation to the viscosity solution, we use the monotone scheme introduced in [1] for unstructured triangular meshes. To verify whether or not the quality of the approximation is acceptable, that is, to see whether or not the constraint (2) is satisfied, we use an approximate version of the rigorous a posteriori error estimate obtained in [2], namely,

$$
\left\|u-u_{h}\right\|_{L^{\infty}(\Omega)} \leq \Phi\left(u_{h}\right),
$$

where $\Phi$ is a suitably defined non-linear functional which, roughly speaking, depends on the residual. The last component is how to use the information of the residual, the above error estimate and the tolerance to generate the mesh. Our method is not based on local mesh refinement; instead, it generates a new mesh at every iteration step. This approach enables us to generate meshes for which the quality constraint (2) is satisfied with optimal complexity. Moreover, in our experience, this seems to work better than the usual local mesh refinement strategies that are usually used in adaptive algorithms.

The rest of the paper is organized as follows. In Section 2, we describe the details of each component of the main features of the method, namely the monotone numerical scheme that we use, the use of approximate a posteriori error estimate, and the way we update the mesh. In Section 3, we carry out a thorough numerical study of its performance on several test problems. Finally, in Section 4 we end with some concluding remarks.

\section{The Adaptive Method}

For any given tolerance $\tau>0$, the adaptive method proceeds as follow:

(0) Construct an initial mesh $\Omega_{h}$.

(1) Compute an approximate solution $u_{h}$ on $\Omega_{h}$.

(2) If $\Phi_{h}\left(u_{h}\right) \leq \tau$ then take $u_{h}$ as the approximation sought and stop.

(3) Otherwise, compute a new mesh $\Omega_{h}$, and go to (1). 
In the rest of this section we describe all these steps in details, namely we explain the numerical method that we use to compute the approximate solution, the approximate a posteriori error estimate $\Phi_{h}\left(u_{h}\right)$, and most importantly how we compute a new mesh. But first, we begin by defining the viscosity solution of the problem under consideration.

\subsection{The viscosity solution}

To state the definition of the viscosity solution of the model equation (1), we need the definition of semi-differentials of a function. We follow the same notation as in [4] for this section and for the section on a posteriori error estimate. The super-differential of a function $u$ at a point $x \in \mathbb{R}^{2}, D^{+} u(x)$, is the set of all vectors $p$ in $\mathbb{R}^{2}$ such that

$$
\limsup _{y \rightarrow x}\left(\frac{u(y)-\{u(x)+(y-x) \cdot p\}}{\|y-x\|}\right) \leq 0,
$$

and the sub-differential of a function $u$ at a point $x \in \mathbb{R}^{2}, D^{-} u(x)$, is the set of all vectors $p$ in $\mathbb{R}^{2}$ such that

$$
\liminf _{y \rightarrow x}\left(\frac{u(y)-\{u(x)+(y-x) \cdot p\}}{\|y-x\|}\right) \geq 0 .
$$

Also we need the following quantity before we define the viscosity solution:

$$
R(u ; x, p)=u(x)+H(p)-f(x),
$$

which is just the residual of $u$ at $x$ if $p=\nabla u(x)$.

Definition 2.1 (The viscosity solution [5]) A viscosity solution of the Hamilton-Jacobi equation (1) is a continuous periodic function on $\mathbb{R}^{2}$ such that, for all $x$ in $\mathbb{R}^{2}$,

$+R(u ; x, p) \leq 0 \quad \forall p \in D^{+} u(x), \quad$ and $\quad-R(u ; x, p) \leq 0 \quad \forall p \in D^{-} u(x)$.

If both the Hamiltonian $H$ and the right-hand side $f$ of (1) are Lipschitz, then the viscosity solution exists. See [5] and the references therein for more general results. 


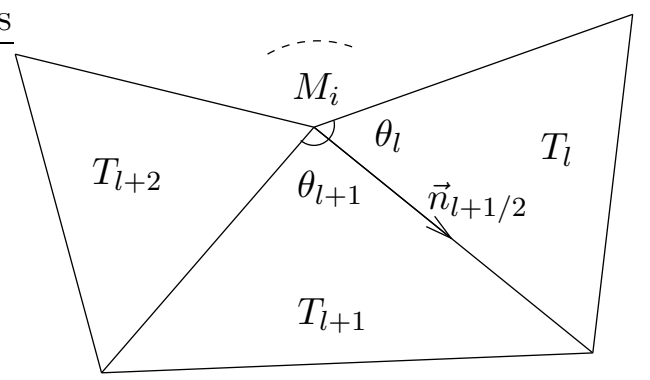

Figure 1: Structure of the triangular mesh around a node $M_{i}$

\section{$2.2 \quad$ A monotone scheme}

We use the Lax-Friedrich's type monotone scheme on triangular meshes introduced by Abgrall [1]. Here we define the numerical scheme, but we leave out its derivation and convergence properties. We refer interested reader to [1] for more details.

Let $T_{h}$ be a triangulation of $(0,1) \mathrm{x}(0,1),\left\{M_{i}\right\}_{i=1}^{n}$ be the set of nodes. For a fixed $i$, let $\left\{T_{l}\right\}_{l=0}^{k_{i}}$ be the set of triangles having $M_{i}$ as a vertex, see Figure 1. To this set of triangles is associated a family of angular sectors $\left\{\mathcal{O}_{l}\right\}_{l=0}^{k_{i}}$ defined as the inner sectors at $M_{i}$ of all the triangles in $\left\{T_{l}\right\}_{l=0}^{k_{i}}$. Let $\theta_{l}$ denote the angle of $\mathcal{O}_{l}$, and let's assume that the indexing of the angular sectors is done clock-wise so that $\theta_{l}>0$, and $\sum_{l=0}^{k_{i}} \theta_{l}=2 \pi$. We denote the unit vector of the half-line $\mathcal{O}_{l} \cap \mathcal{O}_{l+1}$ by $\vec{n}_{l+1 / 2}$, and we define $\beta_{l+1 / 2}$ as

$$
\beta_{l+1 / 2}=\tan \left(\frac{\theta_{l}}{2}\right)+\tan \left(\frac{\theta_{l+1}}{2}\right), \quad l=0, \ldots, k_{i}
$$

where $k_{i}+1$ is associated with 0 . For any function $\phi$ let $\phi_{i}:=\phi\left(M_{i}\right), \quad i=$ $1, \ldots, n$. We consider the function $u$, the solution we seek, to be piecewise linear on $T_{h}$, therefore its gradient is piecewise constant. Let $\nabla u_{T_{l}}$ be the constant value of the gradient of $u$ on the triangle $T_{l}$.

The numerical scheme is defined as follows:

$$
u_{i}+\mathcal{H}\left(u_{i}\right)=f_{i}, \quad i=1, \ldots, n
$$

where

$$
\mathcal{H}\left(u_{i}\right)=H\left(\frac{\sum_{l=0}^{k_{i}} \theta_{l} \nabla u_{T_{l}}}{2 \pi}\right)-\omega \sum_{l=0}^{k_{i}} \beta_{l+1 / 2}\left(\frac{\nabla u_{T_{l}}+\nabla u_{T_{l+1}}}{2}\right) \cdot \vec{n}_{l+1 / 2} .
$$


Here the artificial viscosity term $\omega$ is defined as

$$
\omega=\frac{C(L)}{\pi}
$$

where $C(L)$ is a Lipschitz constant for $H$ on the set $\left\{p \in \mathbb{R}^{2}:\|p\|<1\right\}$.

Since $H$ is non-linear for our test problems, the scheme is implicit. Therefore we use the Newton's Method to solve the non-linear system iteratively. To solve the linear equations, we used the GMRES method as implemented in the package SPARSKIT2, created by Youcef Saad [11].

\subsection{The approximate a posteriori error estimate}

In this subsection, we present the rigorous a posteriori error estimate obtained in [2], and then the approximate a posteriori error estimate that we use. We start by introducing several quantities that we need to state the error estimate. First, we introduce the following semi-norms:

$$
|u-v|_{-}=\sup _{x \in \Omega}(u(x)-v(x))^{+}, \quad|u-v|_{+}=\sup _{x \in \Omega}(v(x)-u(x))^{+},
$$

where $w^{+} \equiv \max \{0, w\}$, and $\Omega=\mathbb{R}^{2}$. We consider $\Omega=(0,1) \mathrm{x}(0,1)$ because of the periodicity.

Then we define a generalization of the residual:

$$
R_{\epsilon}(u ; x, p)=u(x)+H(p)-f(x-\epsilon p)-\frac{1}{2} \epsilon|p|^{2},
$$

which we call the shifted residual.

And finally, we introduce the paraboloid,

$$
P_{v}(x, p, \kappa ; y)=v(x)+(y-x) \cdot p+\frac{\kappa}{2}|y-x|^{2} \quad y \in \mathbb{R}^{2},
$$

where $x$ is a point in $\mathbb{R}^{2}, p$ is a vector of $\mathbb{R}^{2}$, and $\kappa$ is a real number.

The rigorous a posteriori error estimate is stated in the following theorem.

Theorem 2.2 (A posteriori error estimate [2]) Let $u$ be the viscosity solution of the model equation (1) and let $v$ be any continuous function on $\mathbb{R}^{2}$ periodic in each coordinate with period 1 . Then, for $\sigma \in\{-,+\}$,

$$
|u-v|_{\sigma} \leq \inf _{\epsilon \geq 0} \Phi_{\sigma}(v ; \epsilon)
$$


where

$$
\Phi_{\sigma}(v ; \epsilon)=\sup _{(x, p) \in \mathcal{A}_{\sigma}(v ; \epsilon)}\left(\sigma R_{\sigma \epsilon}(v ; x, p)\right)^{+} .
$$

The set $\mathcal{A}_{\sigma}(v ; \epsilon)$ is the set of elements $(x, p)$ satisfying

$$
\begin{aligned}
& x \in \Omega \\
& \sigma\left\{v(y)-P_{v}(x, p, \sigma / \epsilon ; y)\right\} \leq 0 \quad \forall y \in \mathbb{R}^{2} .
\end{aligned}
$$

(For $\epsilon=0$, we only require the first condition and that $p \in D^{\sigma} v(x)$.)

Note that the above result implies that

$$
\|u-v\|_{L^{\infty}(\Omega)} \leq \Phi(v),
$$

where

$$
\Phi(v)=\max \left\{\inf _{\epsilon \geq 0} \Phi_{+}(v, \epsilon), \inf _{\epsilon \geq 0} \Phi_{-}(v, \epsilon)\right\} .
$$

In practice, we do not use the above functional $\Phi$, but an approximation $\Phi_{h}$, also introduced in [2]. The functional $\Phi_{h}$ is given by

$$
\Phi_{h}(v)=\max \left\{\inf _{\epsilon \in \mathcal{E}_{h}} \Phi_{h,+}(v, \epsilon), \inf _{\epsilon \in \mathcal{E}_{h}} \Phi_{h,-}(v, \epsilon)\right\} .
$$

Rather than taking the parameter $\epsilon$ in the interval $[0, \infty)$, we take it in the discrete set $\mathcal{E}_{h}$, where

$$
\mathcal{E}_{h}=\{i \cdot E / N, 0 \leq i \leq N\}
$$

where

$$
E=2 h \omega|\ln (h \omega)| \quad \text { and } \quad N=4|\ln (h \omega)| .
$$

Here $\omega$ is the artificial diffusion coefficient used in the numerical scheme (4), and $h$ is the size of the largest element in the triangulation. This choice of $\mathcal{E}_{h}$ is motivated in [2].

It remains to describe the functional $\Phi_{h, \sigma}(v, \epsilon)$. These functionals are obtained from the functional $\Phi_{\sigma}(v, \epsilon)$ by simply replacing the set $\mathcal{A}_{\sigma}(v ; \epsilon)$ by the set $\mathcal{A}_{h, \sigma}(v ; \epsilon)$ which is defined as the set of elements $(x, p)$ satisfying

$$
\begin{aligned}
& x \in \Omega_{c}, \\
& \sigma\left\{v(y)-P_{v}(x, p, \sigma / \epsilon ; y)\right\} \leq 0 \quad \forall y \in \Omega_{c}:|y-x| \leq 2\|v\|_{\operatorname{Lip}(\Omega)} \epsilon,
\end{aligned}
$$


where $\Omega_{c}$ is the set of barycenters of the elements in the triangulation. Note that since we assume $u_{h}$ to be piecewise linear, when $v=u_{h}$ and $x$ is the center of a triangle $T_{l}$, then the only element in $D^{\sigma} v(x)$ is $p=\nabla u_{h}(x)$, the gradient of $u_{h}$ on the element $T_{l}$. Hence we have a very simple way to both compute the shifted residual, and find the set $\mathcal{A}_{h, \sigma}(v ; \epsilon)$. Note also that if we replace $\Omega_{c}$ by $\Omega$, the above test is equivalent to the original test in Theorem 2.2 ; see $[2]$.

\subsection{Computing a new mesh}

Given a mesh $\Omega_{h}^{k}$, we compute an approximate solution $u_{h}^{k}$ by using the numerical scheme (3). If $\Phi_{h}\left(u_{h}^{k}\right) \geq \tau$, we must compute a new mesh, $\Omega_{h}^{k+1}$. In [4], where we considered the 1-D case, we computed a new grid by properly defining a positive function $\Gamma$ in terms of the old mesh, solving the equation

$$
\frac{1}{\Gamma} \frac{d}{d x} \mathcal{N}=1 \quad \text { in }(0,1), \quad \text { where } \mathcal{N}(0)=0,
$$

and, finally, setting

$$
\left\{x_{j}=\mathcal{N}^{-1}(j)\right\}_{j=1}^{n},
$$

where $n$ is the smallest natural number greater or equal to $\mathcal{N}(1)$. A natural extension of such procedure would be to replace the above boundary value problem with using the eikonal equation

$$
\frac{1}{\Gamma}|\nabla \mathcal{N}|=1 \quad \text { in } \Omega, \quad \text { where } \mathcal{N}=0 \quad \text { on } \partial \Omega .
$$

Mesh-generation algorithms using (more shophisticated versions of) this idea had already been proposed and tested by Hoch and Rascle [8] with very impressive results. To give a rough idea of how to do this, take $\Omega$ to be $\mathbb{R}^{2} \backslash P$. Then

$$
\mathcal{N}(Q)=\int_{P}^{Q} \Gamma \mathrm{d} \gamma
$$

where the integral is evaluated along the segment joining $P$ and $Q$. If $\Gamma=$ $1 / h$, the points $Q$ for which $\mathcal{N}(Q)=1$ form nothing but the circle of radius $h$ centered at the point $P$. Then, six equilateral triangles could be easily formed with one of their vertices being the point $P$. Repeating this process, we could mesh $\mathbb{R}^{2}$ with equilateral triangles. If we take $\Gamma$ equal to $1 / h^{k}$, where the mesh-size function $h^{k}$ is a piecewsie constant function equal to the size of the longest edge of the triangle $T$ of the mesh $\Omega_{h}^{k}$, then we can generate a new mesh with a similar technique. If we want to modify the 
mesh $\Omega_{h}^{k}$ according to the mesh modification function $\mu$, we could take $\Gamma$ equal to $\mu / h^{k}$. In this case, the quantity

$$
l(P, Q)=\int_{P}^{Q} \frac{\mu(s)}{h^{k}(s)} \mathrm{d} \sigma
$$

can be thought of as being a normalized distance $P$ and $Q$ and what we want is to generate a mesh on which the normalized length of each edge of elements is equal to 1 . As an example, consider the case when the function $\mu$ is identically equal to an integer $m$. Then the normalized lengths of the edges of the old mesh, $\Omega_{h}^{k}$, are equal to $m$. Therefore the new mesh should have elements $m$ times smaller than the elements of the old one. Since the function $\mu$ determines how the old mesh is to be modified, it is called the mesh modification function.

In this paper, we do not follow the strategy proposed in [8]. Instead, we use a somewhat related, but different, algorithm which is implemented in the mesh generation package $\mathbf{G m s h}$, created by Christophe Geuzaine and Jean-François Remacle [7]. First, we generate the mesh of the boundary of the domain with the same algorithm used for 1-D problems in [4]. Then, we generate a coarse initial mesh of the domain with no interior points by a constrained Delaunay algorithm [12], which keeps the boundary mesh unchanged. We add new points, namely, the centroid of the elements of the current mesh whose normalized size is bigger than 1 by using the BowyerWatson algorithm [10]. We do this until the normalized lengths of all the edges are less than or equal to one. See [6] for more details on this algorithm as implemented in Gmsh.

It only remains to describe how to define the mesh modification function. We take $\mu(s)=\Psi\left(\gamma^{k}(s)\right)$, where

$$
\gamma^{k}(s)=\frac{1}{\tau} \min \left\{\max _{B_{l}}\left|R_{l_{m}}\right|, \Phi_{h}\left(u_{h}^{k}\right)\right\},
$$

for $s \in T_{l}$, where the set $B_{l}$ consists of the element $T_{l}$, three immediate neighboring elements of $T_{l}$, and the neighboring elements of those three. Here, the value of the residual on any triangle $T_{l_{m}}$ is denoted by $R_{l_{m}}$. Moreover, $\Psi$ is given by,

$$
\Psi(t)= \begin{cases}t(1+\sqrt{t-1}), & t>1 \\ \frac{t+3}{4}, & 0 \leq t \leq 1\end{cases}
$$

See [4] for a thorough discussion of this function. This choice works equally well for both 1-D and 2-D problems as indicated by the numerical results 
in the next section. We use this choice of $\Psi$ after the second step. For the first two steps, we use $\Psi(t)=t$ since, typically, the corresponding mehses we use are very coarse the function $\gamma^{k}$ is already quite large.

If we start the algorithm with a very coarse mesh, then the mesh generated after the first iteration is not regular. This irregularity propagates into the next iterations and affects the quality of the meshes. Therefore we find it helpful to replace the adaptive irregular mesh at the second step with a uniform mesh with the same number of nodes. This improves the quality of the adaptive meshes in the successive iterations, however it does not alter the convergence properties of the method.

\section{$3 \quad$ Numerical Results}

\subsection{The test problems}

We test our algorithm on four test problems; see Table 1. Problem 1 is one of the 1-D problems tested in [4], now extended to 2-D. We consider this problem to see whether or not the one-dimensional mesh structure is actually preserved when we use the two-dimensional version of the adaptive method. Then, to study the performance of the algorithm on two dimensional problems, we consider three other problems. We consider a problem with a smooth solution, problem 2, as well as two problems with kinks in the viscosity solution, problems 3 and 4 . Note that our problem set contains problems with both convex and non-convex Hamiltonians.

Table 1: Test problems.

\begin{tabular}{cccc}
\hline Problem & $H(p)$ & right-hand side $f(x, y)$ & viscosity solution $u(x, y)$ \\
\hline (1) & $-p_{1}^{2} / 4 \pi^{2}$ & $\cos ^{4}(\pi x)$ & $\cos ^{2}(\pi x)$ \\
& $p_{1}^{2}+p_{2}^{2}$ & $\begin{array}{c}(\sin (2 \pi x)+\cos (2 \pi y)) / 2 \pi \\
+\cos ^{2}(2 \pi x)+\sin ^{2}(2 \pi y)\end{array}$ & $(\sin (2 \pi x)+\cos (2 \pi y)) / 2 \pi$ \\
& & $-|\cos (\pi x)|-|\cos (\pi y)|$ & $-|\cos (\pi x)|-|\cos (\pi y)|$ \\
$(\mathbf{2})$ & $\left(p_{1}^{2}+p_{2}^{2}\right) / \pi^{2}$ & $\sin ^{2}(\pi x)+\sin ^{2}(\pi y)$ & \\
& & $\begin{array}{c}u(x, y)-\sin ^{4}(\pi(x+y)) / 4 \\
+\sin ^{2}(\pi(x+y))-1\end{array}$ & $-|\cos (\pi(x+y))| / 2 \pi$ \\
& & &
\end{tabular}




\subsection{Results with the adaptive method}

We begin by testing the quality of the a posteriori error estimate. To do that, we study the behavior of the effectivity index

$$
e i_{h}\left(u, u_{h}\right)=\frac{\Phi_{h}\left(u_{h}\right)}{\left\|u-u_{h}\right\|_{L^{\infty}\left(\Omega_{h}\right)}}
$$

on approximate solution obtained with uniform meshes. In Table 2, we see that, as expected, the order of the scheme is one, and that the a posteriori error estimate is reasonable given that its values remain close to 1 and do not increase, or increase very slowly, when the mesh is refined.

Next, we compare the performance of the adaptive method in the 1-D and the 2-D cases. In Fig. 2, we show the results for the first test problem on Table 1 with $\tau=2.5 \mathrm{E}-2$ for both cases. We see that the results for 2-D case are qualitatively similar to those for 1-D. Indeed, they exhibit a 1-D structure since their main features are essentially constant along any line parallel to the $y$-axis. This gives a strong indication that it is reasonable to expect that the remarkable results obtained for the 1-D case will also hold in the 2-D case, in spite of the use of triangular unstructured meshes and a different algorithm to compute them.

Finally, in Table 3, we display the results obtained by applying the adaptive method to the four test problems. The method is tested for the tolerance values $\tau=10 \mathrm{E}-2,5 \mathrm{E}-2$, and 2.5E-2. We measure the success of the method by considering two effectivity indexes. The first is

$$
e i_{\tau}\left(\tau ; u, u_{h}\right)=\frac{\tau}{\left\|u-u_{h}\right\|_{L^{\infty}\left(\Omega_{h}\right)}},
$$

and shows whether the strict error control is achieved. From Table 3, we see that this index is always bigger than 1 . In ohter words, the method enforces a strict error control and is thus reliable. The second effectivity index we consider is

$$
e i_{a d a p}\left(\tau ; u_{h}\right)=\frac{\tau}{\Phi_{h}\left(u_{h}\right)},
$$

which is a measure of the quality of the adaptive method. We see that this index is remarkably close to the ideal value 1 , in spite of the variation of the value of the tolerance. This is an indication that the method is efficient. To further explore this issue, we need to observe the distribution of the error and the residual on the entire domain. We plot these in Figs. 4 and 5. (In Fig. 3, we show some meshes and the logarithm of their respective mesh size functions to give an idea of the meaning of such plots.) We see that the 
mesh modification function $\Psi(\gamma)$ is very close to the ideal value 1 almost everywhere. This indicates that any further modification of the mesh would result in very insignificant changes.

The above discussion refers only to the last step of the adaptive method. To measure the efficiency of the whole method we need to consider the computational complexity necessary to carry out all the steps. Since, in our case, the computational complexity is proportional to the number of nodes in the mesh, we introduce the quantity

$$
\text { cmplxr }=\frac{\sum_{k=1}^{K} n^{k}}{n^{K}}
$$

where $n^{k}$ is the number of nodes of the mesh of the step $k$, and $K$ is the number of steps needed for convergence. We use this quantity to compare the computational complexity needed to carry out all the steps of the method to the computational complexity needed to carry out the last one. A bigger complexity ratio indicates a less efficient adaptive method. In the last two columns of Table 3, we display the numbers of steps needed for convergence and the complexity ratio. We see that the complexity ratio is always less than 4 , independently of the problem and the value of the tolerance. In other words, the observed complexity of the method is optimal.

\subsection{Adaptivity versus uniform refinement}

In this subsection we compare the performance of our adaptive method and that of using uniform meshes. In all our computations uniform triangular meshes are generated by drawing the diagonals of uniform rectangular meshes.

In Fig. 6, we make this comparison by considering both the exact error $\left\|u-u_{h}\right\|_{L^{\infty}\left(\Omega_{h}\right)}$ and the approximate a posteriori error estimate, $\Phi_{h}\left(u_{h}\right)$. We see that the adaptive method is more efficient in all the cases. For Problem 3, when considering the exact error for large tolerances both methods seem to perform similarly. However, when the tolerance becomes smaller the adaptive method becomes more efficient. This slight disadvantage at the beginning is actually compensated by a significant success when considering the approximate a posteriori error estimate. Hence over all the adaptive method is considerably more efficient. 
Table 2: History of convergence for uniform grids.

\begin{tabular}{|c|c|c|c|c|}
\hline Problem & $n$ & $\left\|u-u_{h}\right\|_{L^{\infty}\left(\Omega_{h}\right)}$ & order & $e i_{h}\left(u, u_{h}\right)$ \\
\hline \multirow[t]{7}{*}{ (1) } & $20^{2}$ & $9.3 \mathrm{E}-2$ & 0.00 & 1.08 \\
\hline & $40^{2}$ & $4.8 \mathrm{E}-2$ & 0.94 & 1.05 \\
\hline & $80^{2}$ & $2.5 \mathrm{E}-2$ & 0.98 & 1.04 \\
\hline & $160^{2}$ & $1.2 \mathrm{E}-2$ & 0.99 & 1.03 \\
\hline & $320^{2}$ & $6.2 \mathrm{E}-3$ & 0.99 & 1.03 \\
\hline & $640^{2}$ & $3.1 \mathrm{E}-3$ & 1.00 & 1.03 \\
\hline & $1280^{2}$ & $1.6 \mathrm{E}-3$ & 1.00 & 1.03 \\
\hline \multirow[t]{7}{*}{ (2) } & $20^{2}$ & $3.8 \mathrm{E}-1$ & 0.00 & 1.82 \\
\hline & $40^{2}$ & $1.9 \mathrm{E}-1$ & 0.96 & 1.92 \\
\hline & $80^{2}$ & $1.0 \mathrm{E}-1$ & 0.98 & 1.95 \\
\hline & $160^{2}$ & $5.0 \mathrm{E}-2$ & 0.99 & 1.92 \\
\hline & $320^{2}$ & $2.5 \mathrm{E}-2$ & 0.99 & 1.86 \\
\hline & $640^{2}$ & $1.2 \mathrm{E}-2$ & 0.99 & 1.80 \\
\hline & $1280^{2}$ & $6.0 \mathrm{E}-3$ & 1.00 & 1.75 \\
\hline \multirow[t]{7}{*}{ (3) } & $20^{2}$ & $3.8 \mathrm{E}-1$ & 0.00 & 1.46 \\
\hline & $40^{2}$ & $1.9 \mathrm{E}-1$ & 0.97 & 1.46 \\
\hline & $80^{2}$ & $1.0 \mathrm{E}-1$ & 0.98 & 1.67 \\
\hline & $160^{2}$ & $5.0 \mathrm{E}-2$ & 0.99 & 2.11 \\
\hline & $320^{2}$ & $2.5 \mathrm{E}-2$ & 0.99 & 2.13 \\
\hline & $640^{2}$ & $1.2 \mathrm{E}-2$ & 1.00 & 2.15 \\
\hline & $1280^{2}$ & $6.0 \mathrm{E}-3$ & 1.00 & 2.60 \\
\hline \multirow[t]{7}{*}{ (4) } & $20^{2}$ & $2.3 \mathrm{E}-1$ & 0.00 & 1.07 \\
\hline & $40^{2}$ & $1.2 \mathrm{E}-1$ & 0.92 & 1.91 \\
\hline & $80^{2}$ & $6.1 \mathrm{E}-2$ & 0.97 & 1.55 \\
\hline & $160^{2}$ & $3.1 \mathrm{E}-2$ & 0.98 & 1.96 \\
\hline & $320^{2}$ & $1.6 \mathrm{E}-2$ & 0.99 & 2.52 \\
\hline & $640^{2}$ & $7.8 \mathrm{E}-3$ & 1.00 & 3.13 \\
\hline & $1280^{2}$ & $3.9 \mathrm{E}-3$ & 1.00 & 3.35 \\
\hline
\end{tabular}




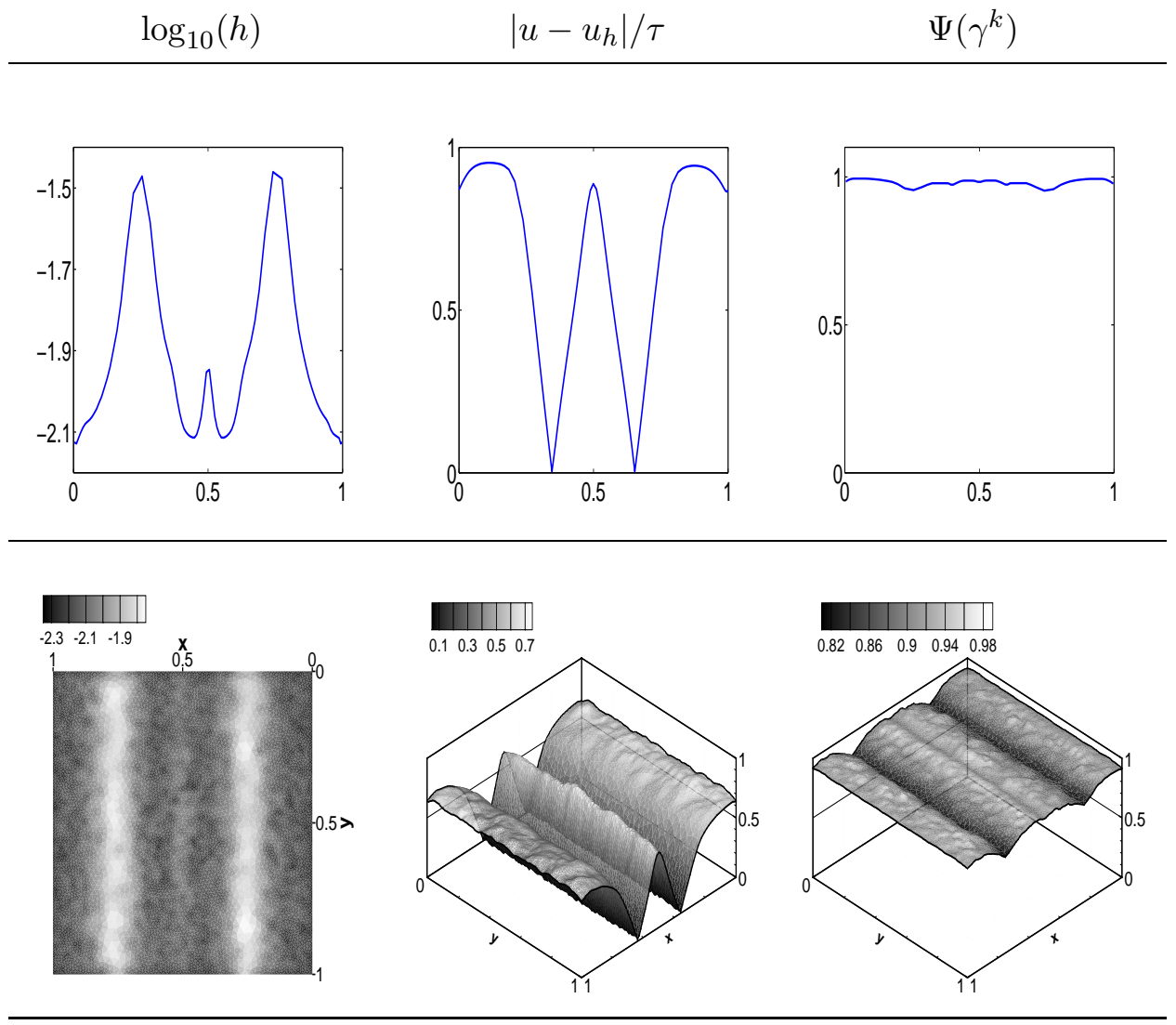

Figure 2: The final step of the Problem 1 for $\tau=2.5 E-2$ : One- (top) and two-dimensional (bottom) algoritms. 

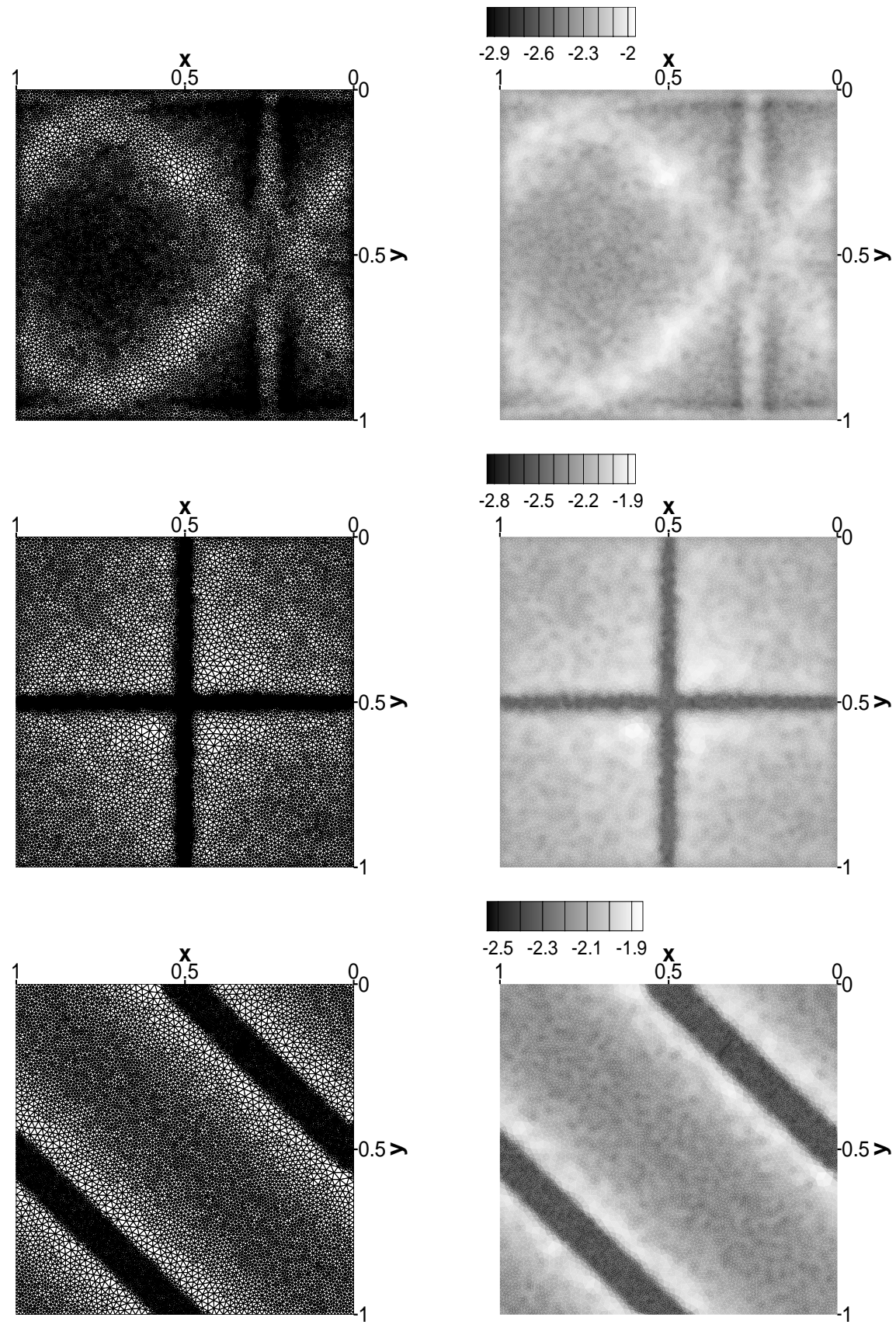

Figure 3: Actual adaptive meshes (left column) and their mesh-size functions $\log _{10}(h)$ (right column) for Problems 2 (top), 3 (middle), and 4 (bottom). 


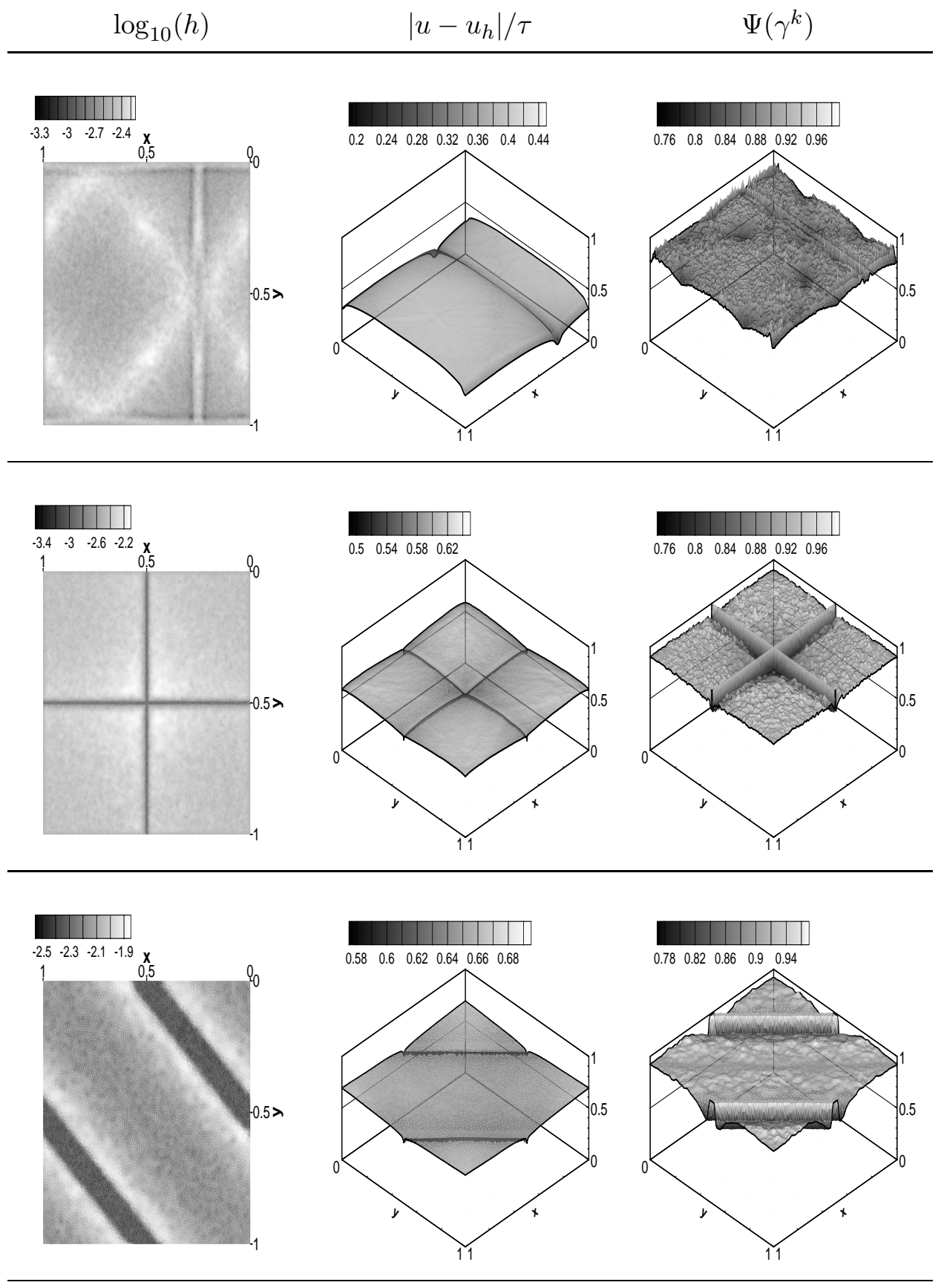

Figure 4: The final step for $\tau=5.0 \mathrm{E}-2$, for Problem 2 (top), Problem 3 (center), and Problem 4 (bottom). 


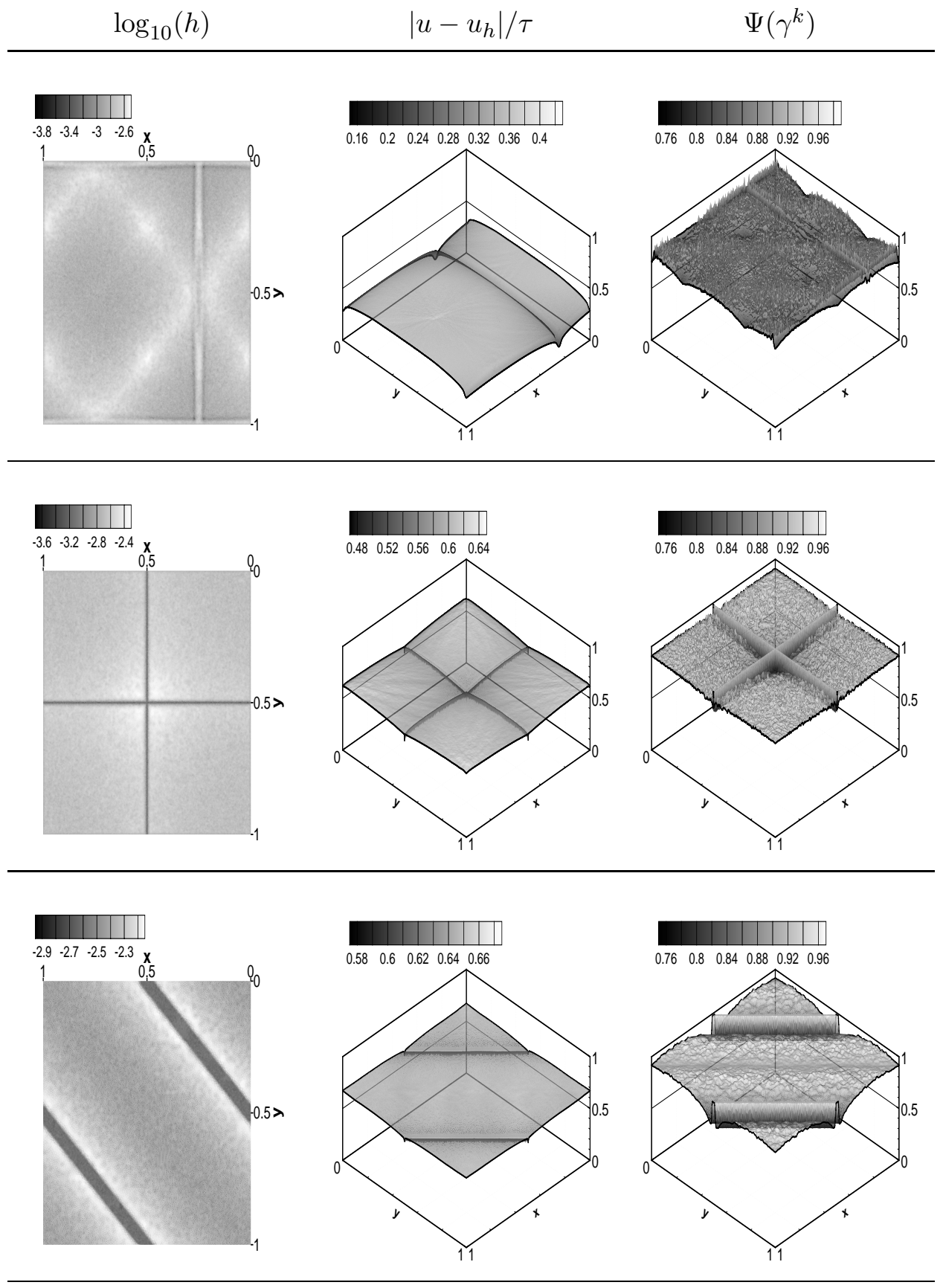

Figure 5: The final step for $\tau=2.5 \mathrm{E}-2$, for Problem 2 (top), Problem 3 (center), and Problem 4 (bottom). 

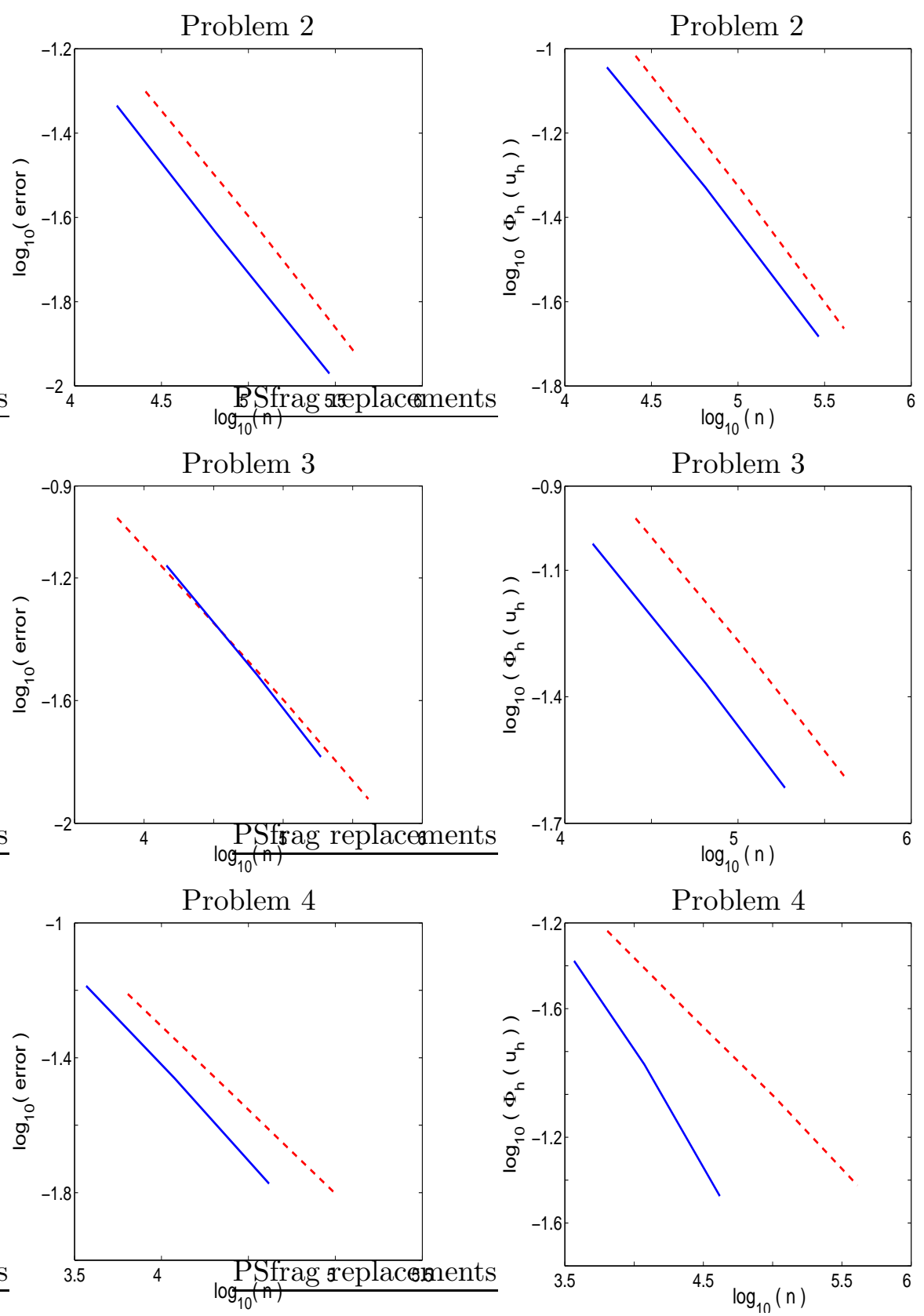

Figure 6: Comparison of convergence rates: Uniform (dashed line) and adaptive (solid line) refinement. 
Table 3: Results of the adaptive method.

\begin{tabular}{ccccccccc}
\hline Problem & $\tau$ & $n$ & $\left\|u-u_{h}\right\|_{L}{ }_{\left(\Omega_{h}\right)}$ & order & ei $i_{\tau}$ & eiadap & steps & cmplxr \\
\hline \multirow{2}{*}{$(\mathbf{1})$} & $10.0 \mathrm{E}-2$ & 392 & $8.2 \mathrm{E}-2$ & - & 1.20 & 1.01 & 3 & 1.96 \\
& $5.0 \mathrm{E}-2$ & 1475 & $4.1 \mathrm{E}-2$ & 1.06 & 1.21 & 1.00 & 3 & 1.82 \\
& $2.5 \mathrm{E}-2$ & 7032 & $1.9 \mathrm{E}-2$ & 1.01 & 1.34 & 1.06 & 4 & 2.43 \\
\hline \multirow{2}{*}{$(\mathbf{2})$} & $10.0 \mathrm{E}-2$ & 17499 & $4.6 \mathrm{E}-2$ & - & 2.16 & 1.10 & 6 & 3.78 \\
& $5.0 \mathrm{E}-2$ & 64830 & $2.3 \mathrm{E}-2$ & 1.06 & 2.16 & 1.06 & 5 & 2.99 \\
& $2.5 \mathrm{E}-2$ & 292324 & $1.0 \mathrm{E}-2$ & 1.02 & 2.33 & 1.20 & 5 & 2.84 \\
\hline \multirow{2}{*}{$(3)$} & $10.0 \mathrm{E}-2$ & 14483 & $6.9 \mathrm{E}-2$ & - & 1.44 & 1.08 & 4 & 2.23 \\
& $5.0 \mathrm{E}-2$ & 65241 & $3.0 \mathrm{E}-2$ & 1.09 & 1.64 & 1.16 & 5 & 2.60 \\
& $2.5 \mathrm{E}-2$ & 186660 & $1.6 \mathrm{E}-2$ & 1.16 & 1.51 & 1.03 & 4 & 2.30 \\
\hline \multirow{2}{*}{$(4)$} & $10.0 \mathrm{E}-2$ & 3690 & $6.5 \mathrm{E}-2$ & - & 1.53 & 1.22 & 4 & 2.03 \\
& $5.0 \mathrm{E}-2$ & 11863 & $3.4 \mathrm{E}-2$ & 1.07 & 1.44 & 1.07 & 3 & 1.42 \\
& $2.5 \mathrm{E}-2$ & 41569 & $1.7 \mathrm{E}-2$ & 1.14 & 1.48 & 1.08 & 3 & 1.66 \\
\hline
\end{tabular}

\section{Conclusions}

In this paper, we have extended the adaptive method proposed in [4] to two dimensional steady-state Hamilton-Jacobi equations. The method has been shown to guarantee a rigorous error control and to be extremely reliable and efficient for a wide variation of the tolerance parameter even in the presence of kinks in the viscosity solution with non-convex Hamiltonians. From these results, it is reasonable to expect that the extension of the algorithm to higher dimensional problems will display a similar performance.

In the time-dependent case, straightforward extensions of the monotone scheme (3) can be used. However, in this case the time-space grids must be allowed to vary locally and the schemes must be properly modified to handle them. The a posteriori error estimate obtained in [3] can be used. Although we have focused on monotone schemes, the adaptive method proposed here can be easily applied to high-order accurate methods like the discontinuous Galerkin method developed in [9]. The above extensions constitute the subject of ongoing work.

Acknowledgment. The authors would like to give special thanks to Christophe Geuzaine for providing a source code for $\mathbf{G m s h}$ and for his help for the installation and execution of the code. 


\section{References}

[1] R. Abgrall, Numerical discretization of the first-order Hamilton-Jacobi equations on triangular meshes, Comm. Pure Appl. Math. 49 (1996), 1339-1377.

[2] S. Albert, B. Cockburn, D. French, and T. Peterson, A posteriori error estimates for general numerical methods for Hamilton-Jacobi equations. Part I: The steady state case, Math. Comp. 71 (2002), 49-76.

[3] _ A posteriori error estimates for general numerical methods for Hamilton-Jacobi equations. Part II: The time-dependent case, Finite Volumes for Complex Applications (R. Herbin and D. Kröner, eds.), vol. III, Hermes Penton Science, June 2002, pp. 17-24.

[4] B. Cockburn and B. Yenikaya, An adaptive method with rigorous error control for the Hamilton-Jacobi equations. Part I: The one-dimensional steady state case, Appl. Numer. Math., to appear.

[5] M. G. Crandall, L. C. Evans, and P. L. Lions, Some properties of viscosity solutions of Hamilton-Jacobi equations, Trans. Amer. Math. Soc. 282 (1984), 487-502.

[6] P.L. George, Tet meshing: Construction, optimization, and adaptation, Proc. 8th International Meshing Rountable, 1999, pp. 133-141.

[7] C. Geuzaine and J. F. Remacle, Gmsh reference manual: the documentation for Gmsh, a finite element mesh generator with built-in pre- and post-processing facilities, URL http://www.geuz.org/gmsh/.

[8] PH. Hoch and M. Rascle, Hamilton-Jacobi equations on a manifold and applications to grid generation or refinement, SIAM J. Math. Anal. 23 (2002), 2055-2073.

[9] C. Hu and C. W. Shu, A discontinuous Galerkin finite element method for Hamilton-Jacobi equations, SIAM J. Sci. Comput. 21 (1999), 666690 .

[10] S. Rebay, Efficient unstructured mesh generation by means of delaunay triangulation and bowyer-watson algorithm, J. Comput. Phys. 106 (1993), no. 1, 125-138.

[11] Y. Saad, Sparskit: a basic tool kit for sparse matrix computations, URL http://www-users.cs.umn.edu/ ${ }^{\text {saad/software/SPARSKIT. }}$ 
[12] J.R. Shewchuk, Constraint delaunay tetrahedralization and provabily good boundary recovery, Proc. 11th International Meshing Roundtable (2002), 193-204. 